\title{
Sapphire, BGO and LiF scintillating bolometers developed for dark matter experiments
}

\author{
N. Coron, J. Gironnet, J. Leblanc, P. de Marcillac, T. Redon \\ Institut d'Astrophysique Spatiale, Bât 121, 91405 Orsay, Paris, France \\ E-mail: noel.coron@ias.u-psud.fr, \\ johann.gironnet@psi.ch, jacques.leblanceias.u-psud.fr, \\ pierre.demarcillac@ias.u-psud.fr, thierry.redon@ias.u-psud.fr
}

\section{E. García, M. Martínez, Y. Ortigoza*, A. Ortiz de Solórzano, C. Pobes, J. Puimedón,} M.L. Sarsa, L. Torres, J.A. Villar

Laboratorio de Física Nuclear y Astropartículas, Universidad de Zaragoza, 50009 Zaragoza,

Spain

E-mail: edgarcia@unizar.es, mariam@unizar.es, ortigoza@unizar.es, alfortiz@unizar.es, cpobes@unizar.es, puimedon@unizar.es, mlsarsa@unizar.es, lidiateunizar.es, villareunizar.es

\begin{abstract}
ROSEBUD (Rare Objects Search with Bolometers UndergrounD) is a collaboration focused on the development of scintillating bolometers for WIMP (Weakly Interacting Massive Particles) searches. In this work we present the results of tests performed with three small scintillating bolometers (50 g sapphire, $46 \mathrm{~g}$ BGO and $33 \mathrm{~g} \mathrm{LiF}$ ) used simultaneously in the same set-up and external background conditions at the Canfranc Underground Laboratory (LSC). Sapphire and BGO have been studied as possible dark matter targets. We analyze their particle discrimination capability with special stress in the discrimination between nuclear recoils and $\beta / \gamma$ background events at low energy. Nuclear recoil spectra obtained simultaneously in both detectors are shown and discussed. The BGO bolometer has also been used as a $\gamma$-ray spectrometer (profiting from its high atomic number content) to analyze and control external $\beta / \gamma$ background in the experiment. $\mathrm{LiF}$ scintillating bolometers could be applied through the ${ }^{6} \mathrm{Li}(\mathrm{n}, \alpha)$ reaction to monitor neutron flux inside the experimental set-up.
\end{abstract}

Identification of dark matter 2008

August 18-22, 2008

Stockholm, Sweden

\footnotetext{
*Speaker.
} 


\section{Introduction}

ROSEBUD is a collaboration between the Institut d'Astrophysique Spatiale (IAS, Orsay, France) and the University of Zaragoza (Zaragoza, Spain), aimed at the direct search for galactic WIMPs with cryogenic detectors at the Canfranc Underground Laboratory (at a depth of $2450 \mathrm{~m}$ of water equivalent). The main objective of ROSEBUD is the development of scintillating bolometers. This hybrid detection technique based on the simultaneous measurement of light and heat allows to discriminate nuclear recoils (like those produced by neutrons or hypothetical WIMPs) from electron recoils (like those produced by $\beta / \gamma$ background) increasing sensitivity for WIMP searches.

In 2007 we performed several tests at the LSC with three small scintillating bolometers: $50 \mathrm{~g}$ sapphire, $46 \mathrm{~g}$ BGO and $33 \mathrm{~g} \mathrm{LiF}$. The three detectors were installed in a common set-up in an ultralow background environment and were cooled down to $20 \mathrm{mK}$ with a small dilution refrigerator. Each detector was mounted in a double bolometer configuration consisting of a scintillating crystal and a Germanium disk inside a reflecting cavity. When a particle interacts in the scintillating crystal, the heat produced is directly measured as a temperature increase and the light is measured through the thermal increase produced in the Ge disk by the scintillation photons eventually absorbed in it. For details on the experimental set-up (dilution refrigerator, double bolometer configuration, electronics and read-out, and internal and external shieldings) see references $[1,2,3]$.

\section{Results and discussion}

The relative light outputs of electron to nuclear recoils (approximately 17 for sapphire [3] and 12 for BGO [4]) have been estimated in dedicated ${ }^{252} \mathrm{Cf}$ neutron source calibrations performed first at Orsay and then in situ at LSC. These high relative light output values allow particle discrimination down to 8.7(15.0) $\mathrm{keV}$ in sapphire and 23.5(33.3) $\mathrm{keV}$ in BGO, with 90\% acceptance of nuclear recoils and $90 \%(99.9 \%)$ rejection of $\beta / \gamma$ events.

Table 1: Rates of nuclear recoils band events measured in sapphire and BGO in different energy windows .

\begin{tabular}{ccc}
\hline $\begin{array}{c}\text { Energy window } \\
(\mathrm{keV})\end{array}$ & $\begin{array}{c}\text { Rate in } \mathrm{Al}_{2} \mathrm{O}_{3} \\
\text { counts/(keV.kg.d) }\end{array}$ & $\begin{array}{c}\text { Rate in BGO } \\
\text { counts/(keV.kg.d) }\end{array}$ \\
\hline $25-50$ & $9.3 \pm 1.8$ & $14.3 \pm 2.5$ \\
$50-100$ & $3.15 \pm 0.76$ & $1.34 \pm 0.55$ \\
$100-200$ & $1.85 \pm 0.41$ & $0.22 \pm 0.16$ \\
$50-200$ & $2.28 \pm 0.38$ & $0.60 \pm 0.21$ \\
\hline
\end{tabular}

Figure 1 displays the low energy spectra of events in the nuclear recoils acceptance band obtained from simultaneous background measurement with sapphire and BGO for a total live time of 51.9 hours. A loss in response in the optical bolometer of the sapphire at LSC forced us to set its discrimination threshold energy at $25 \mathrm{keV}$. The background levels obtained in both bolometers (summarized in Table 1) are very high for being due to neutrons in a deep underground location, and suggest other possible sources for these, at least apparently, non-scintillating events (for example: 

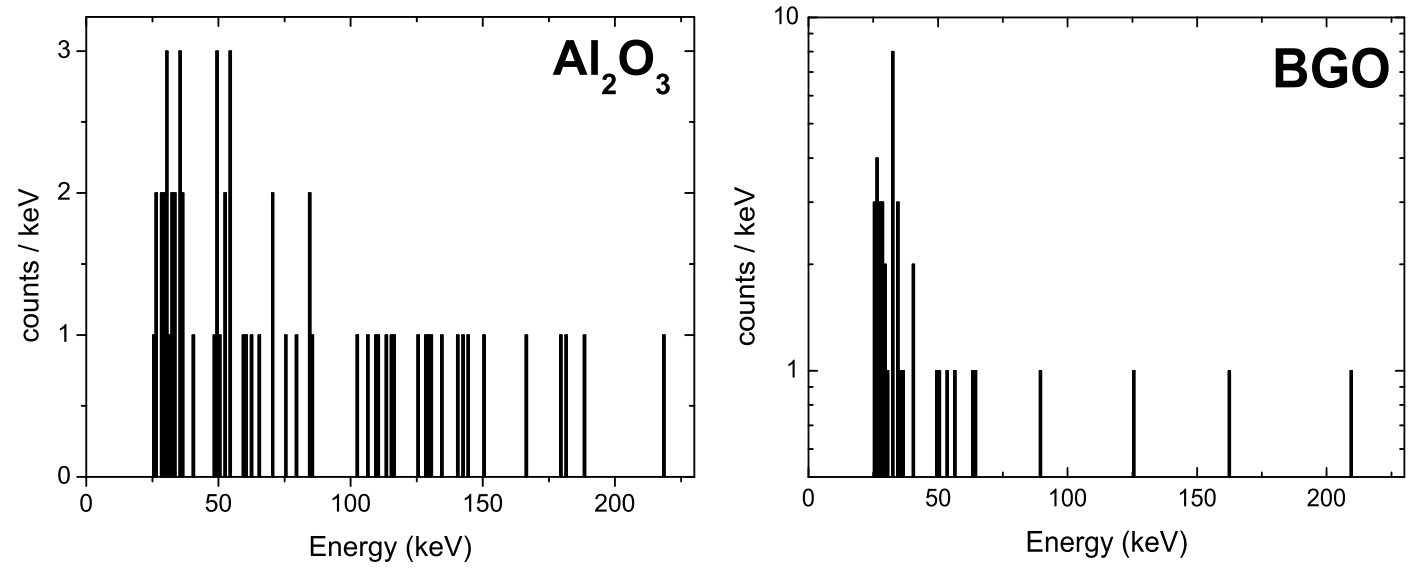

Figure 1: Spectra of events in the nuclear recoils band obtained from simultaneous measurement with sapphire and BGO at LSC (live time $=51.9$ hours). Energy has been estimated from the heat signal.

mechanical cracks, dead volumes, interactions produced in non-scintillating parts attached to the crystals, nuclear recoils produced by alpha contaminations in the surface of the crystals where the alpha particle escapes from the crystal). Further research on the origin of these events is in progress. Future runs will focus on the improvement of the background and discrimination energy threshold.
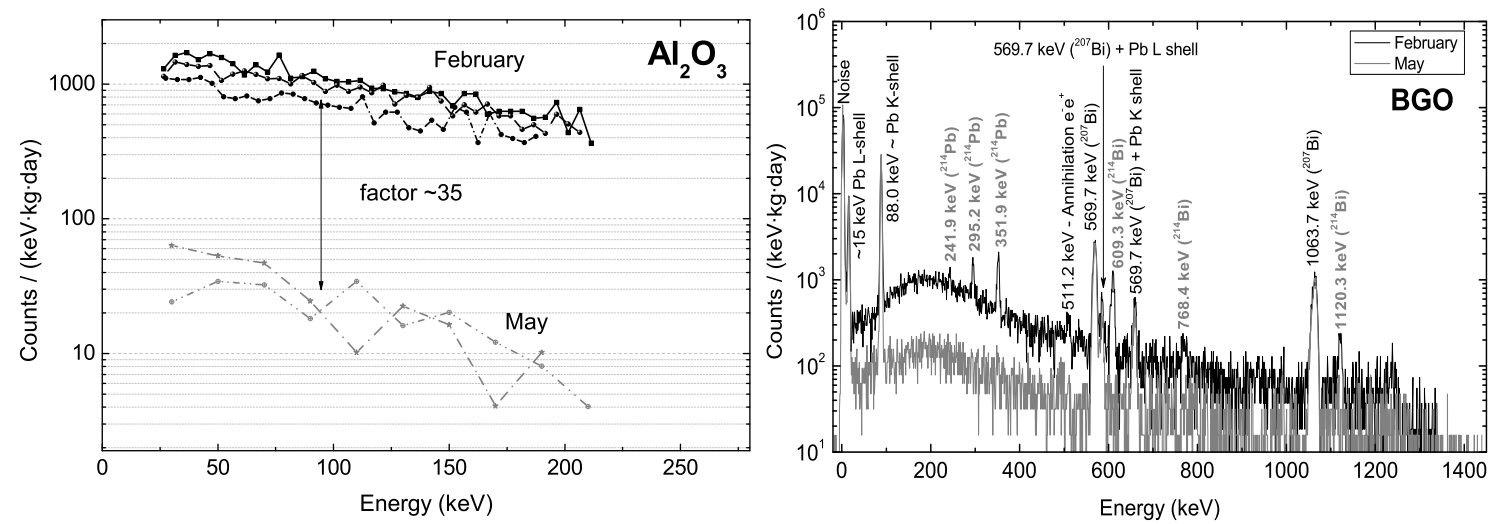

Figure 2: $\beta / \gamma$ spectra of February and May 2007 obtained from simultaneous measurements with the sapphire and BGO at LSC. A reduction of a factor $\sim 35$ from February to May is obtained in all the energy range of sapphire spectra. The spectrum of BGO evidences that this improvement is due to airborne radon removal (peaks arising from ${ }^{222} \mathrm{Rn}$ daughters, labelled in grey, have disappeared in the spectrum of May).

The BGO bolometer, profiting from the presence of bismuth (high atomic number), has also been used as a gamma-ray spectrometer to monitor the external gamma background in the experiment. This capability is illustrated in figure 2 where $\beta / \gamma$ backgrounds measured by the sapphire 
and BGO bolometers are shown.

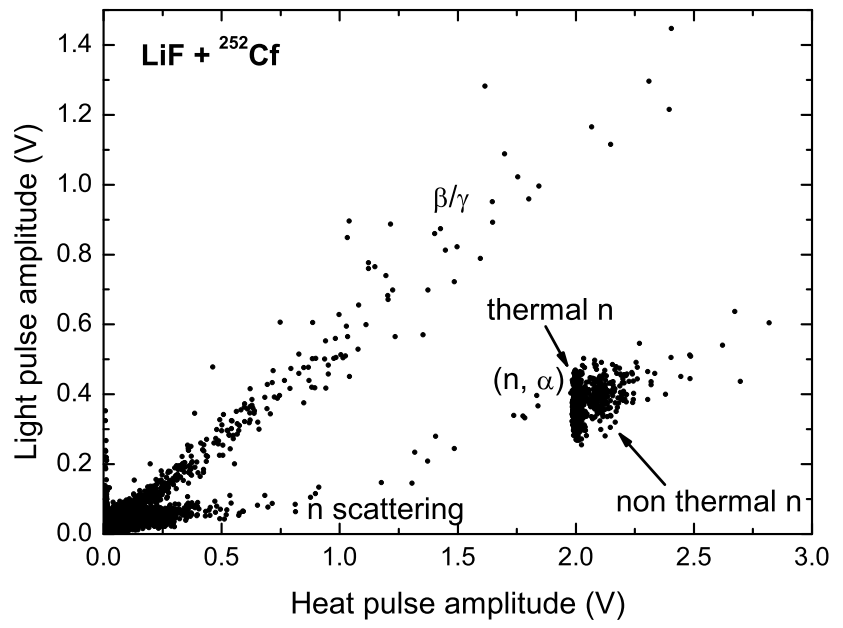

Figure 3: Light versus heat plot obtained in the $33 \mathrm{~g} \mathrm{LiF}$ in a calibration with a ${ }^{252} \mathrm{Cf}$ neutron source at LSC. Neutron capture $(\mathrm{n}, \alpha)$ reaction in ${ }^{6} \mathrm{Li}(\mathrm{Q}$-value $=4.78 \mathrm{MeV})$ allows to monitor both thermal and non-thermal neutrons.

The LiF scintillating bolometer can be used to detect neutrons through the $(\mathrm{n}, \alpha)$ capture reaction in ${ }^{6} \mathrm{Li}$. Events produced by thermal and non-thermal neutrons arising from a ${ }^{252} \mathrm{Cf}$ neutron source are easily identified in the light versus heat signal plot (see figure 3). This preliminary test evidences that monitoring of the environmental neutron flux inside the experimental set-up is feasible with $\mathrm{LiF}$, but larger masses and/or enrichment in ${ }^{6} \mathrm{Li}$ are required.

\section{Acknowledgements}

This work has been partially supported by the French CNRS/INSU (MANOLIA and BOLERO projects), by the Spanish Commission for Science and Technology (MEC, Grants No. FPA200400974 and FPA2007-63777), by the Gobierno de Aragón (Group in Nuclear and Astroparticle Physics) and by the EU Project ILIAS Contract No. RII3-CT-2004-506222. Y. Ortigoza was supported by a UZ/BSCH/Fundación Carolina grant.

\section{References}

[1] S. Cebrián et al. Astrop. Phys. 21 (2004) 23.

[2] N. Coron et al. Opt. Eng. 43 (2004) 1568.

[3] A. Calleja et al. J. Low. Temp. Phys. 151 (2008) 848.

[4] N. Coron et al. Nucl. Instrum. and Methods A 520 (2004) 159. 